\title{
Big Problem or Not a Big Problem: Problems to Health Care Utilization by Residence in The Gambia Among Women of Reproductive Age. A Cross Sectional Study
}

Ebrima Barrow ( $\square$ eboybarrow@gmail.com )

Ministry of Health and Social Welfare

Alieu Sowe

Ministry of Health and Social Welfare

\section{Research Article}

Keywords: permission, money, distance, not wanting to go alone

Posted Date: December 1st, 2021

DOI: https://doi.org/10.21203/rs.3.rs-1068671/v1

License: (c) (i) This work is licensed under a Creative Commons Attribution 4.0 International License.

Read Full License 


\section{Abstract}

Background: Ability to utilise healthcare services is desire for everyone in need. Unfortunately, challenges to health care utilization persist and they do so inequitably amongst social groups. This study aimed to examine problems to health care utilization and residential area equity in utilization among women of reproductive age in The Gambia.

Methods: Data from The Gambia 2019-20 Demographic and Health Survey comprising of 11,865 women $15-49$ years of age was used. A systematic selection method of equal probability was employed for the data collection interviews. Visiting any health facility in the last 12 months is the outcome. Problems to health care utilization was assessed using four primary factors of interest: permission to go, money needed for treatment, distance to health facility and not wanting to go alone as independent variables. Descriptive and logistic regression analysis were used to assess the frequency distribution and the association of the four factors and health care utilization by residence. The point estimates were reported in odds (OR), 95\% confidence interval $(\mathrm{Cl})$ and $p$ value $<0.05$ signified statistical significance.

Results: Most women in rural (79\%) and urban (83\%) residential areas utilized health care services in the last 12 months. Women in urban areas who had a big problem getting permission to go and a big problem not wanting to go alone had lower odds of health care utilization compared to women who did not have a big problem getting permission to go and not wanting to go alone for health care. Women resident in rural areas who reported distance to health facility as a big problem had higher odds of health care utilization compared to women who did not have big problem concerning distance to health facility.

Conclusions: Permission to go and not wanting to go alone appear to be associated with cultural norms and inadequate social support for women utilizing health care in urban residence. Policy makers should address harmful cultural norms and inadequate social support for women during health care visits to improve equity in health care utilization towards achieving universal health coverage.

\section{Introduction}

Health care utilization is the quantification or description of the use of preventive or curative health services to promote ones health and well-being or to obtain information about their health status (1). Health care utilization is influenced by many factors, which can be categorised into predisposing factors, enabling factors, and the need for care (2). These factors can be facilitators or barriers to the used of health care. The socio-cultural beliefs concerning our individual health and the health care system, and demographic characteristics which influence ones use of health are predisposing factors to health care utilization. The opportunities at individual and societal level available for people to utilize health care are enabling factors for health care utilization. The immediate cause prompting the use of health care to improve one's health and wellbeing or obtaining information on one's own health are the need factors to health care utilization (3). Utilization of health care when the need arises is desired by everyone, 
unfortunately challenges to health care utilization led to an increasing gap in healthcare utilization between different social groups by gender or residence.

In many countries, political commitment to improve access to sexual and reproductive health care is becoming an agenda in policy development to improve gender equality. Since the United Nations sustainable development goals (SDG) launched in 2015 is endorsed by many countries, holding countries responsible for providing essential health services to all its population is crucial to its implementation. However, progress on achieving the set targets vary across regions and countries (4). An assessment on the progress of 183 countries towards universal health coverage (UHC), reported a median health service coverage value of $65 \%$ with The Gambia achieving $46 \%$. In these 183 countries the estimated median of health service coverage index is found to be lowest among the poorest population which is evidence of the inequity in health care within national populations (5). In order to close these gaps and achieve universal health coverage SGD 3.7 and 3.8 targets, there is a need for government and development partner commitment to the implementation of the United Nations Global health policies to provide universal access to sexual and reproductive health care services (6).

Financial, cultural and geographical location of services are some of the barriers depriving women from utilising healthcare services(7). Data from 57 countries showed that $55 \%$ of women have body autonomy meaning 1 in 2 women have the authority to make their own decision on health care such as when to used health care, including sexual and reproductive health services, when to have a child and when to have sex with their partner (8). Permission to seek for healthcare services attributable to cultural and societal norms place women in disadvantage positions in decision making. These cultures and societal norms have consequences on women's health seeking behaviour and health care access. Women who had no cultural norms which prevent them from accessing health care are 2 times more likely to utilize health care than those that have cultural barriers preventing them from utilizing health care (9). Decision making on health care seeking of women is primarily dominated by men which could be delayed due to partners financial constrain. As a result, health complications may arise from delay in care seeking (10).

Geographical accessibility motivates health care utilization albeit, distance to health facility and lack of transport is one of the most common challenge rural women encounter for antenatal care and health facility delivery (11). Coverage of reproductive health services is lowest for women living in poverty and/or in rural residence therefore ensuring equitable utilization of health care base on need requires distribution of health services to low income and rural populations (5). For countries such as The Gambia, Ghana and Kenya that have introduced free maternal and child health services, success of this policy will only be attained if health service is accessible to women in hard-to-reach geographical areas. Women in hard to reach area always have to make trade-off between incurring prohibitive cost to travel for health care and none utilization of health care (12). This is indicative of the importance of geographical access to health care use. Companionship or not wanting to go alone during health care seeking provides social support for women in need of care. Not wanting to go alone is reported as a perceived barrier to health care utilization by $10 \%$ of women in The Gambia and by $15 \%$ of women in Ghana $(13,14)$. 
There has been no known study in The Gambia examining problems to health care utilization by residence and the association of these problems to health care utilization by residence. Therefore, targeting women of reproductive age in this study will produce important findings essential to reduce the barriers to health care utilization for women. Consequently, utilizing essential health care will improve maternal and child health indicators in The Gambia thereby accelerating progress towards universal health coverage.

\section{Aims}

This study aims to examine problems to health care utilization by residence among women of reproductive age in The Gambia. Findings from this study will compliment evidence on health care utilization and inequity by residence and problems associated with health care used. Moreover, findings from this study may guide inform policy development on equity and health care utilization.

\section{Research Questions}

1. Is 'permission to go' a problem to health care utilization for women in The Gambia by residence?

2. Is 'money needed for treatment' a problem to health care utilization for women in The Gambia by residence?

3. Is 'distance to health facility' a problem to health care utilization for women in The Gambia by residence?

4. Is 'not wanting to go alone' a problem to health care utilization for women in The Gambia by residence?

\section{Methods}

\section{Setting}

The Gambia is the smallest country in mainland Africa. It covers a length of $400 \mathrm{~km}$ from the east to the west and is about 24 - $50 \mathrm{~km}$ wide. The country covers a land area of about 10,689 square kilometres and is surrounded by the Republic of Senegal on all sides except on the west which is covered by the Atlantic Ocean. The population of The Gambia is estimated to be 1.9 million with a $3.3 \%$ growth rate according to the 2003 population and housing census (13). The Gambia has a population density of 176 people per square kilometres with rising urbanization, nearly $60 \%$ of the population currently live-in urban residence. The Gambia is experiencing a high fertility rate of 5.4 births per woman resulting in $42 \%$ of the country population below 15 years ages of age. The proportion of young people $15-24$ years of age is $22 \%$ of the population and women comprise a proportion of $51 \%$ of the population. Life expectancy is 63.4 years at birth, 62.5 and 65 years for males and females, respectively (13). The market-based economy of The Gambia is mainly subsistence agriculture and tourism, with an estimated Gross Domestic Product (GDP) of $\$ 944$ million. The economy is mainly supported by Agriculture contributing about $22 \%$ of the GDP in 2012 and 2013. Agriculture constitutes $70 \%$ of the country's labour force. Administratively the country is divided into 7 regions including 2 municipalities. During the 2019 - 20 Gambia Demographic and Health 
Survey the country was divided into 8 regions: Banjul, Kanifing, Kerewan, Mansakonko, Kuntaur, Janjabureh, Basse and Brikama (15).

\section{Data source}

This study used data from the Demographic and Health Survey (DHS) conducted in The Gambia in 2019 - 20 (16). The 2019 - 20 Gambia DHS collected household data on key population and health indicators from 7,025 households in 281 sampling units across the country. To arrive at a representative sample of the Gambian population, the following sampling strategies were used. First, 281 main sampling units of settlements was selected initially using a probability proportional to size selection method. Following the selection of these sampling units, a comprehensive listing of households was conducted in every sampling unit. In the second stage, 25 households were selected from the household list of the selected sampling units using an equal probability systematic selection method (15). To control for bias during the data collection, no replacement of the pre-selected households was allowed. For the study precisions to be comparable, 173 clusters of settlement were selected in urban areas and 108 clusters of settlements were selected in rural areas (15) .

Study sample

The 2019 - 20 Gambia DHS includes data collected from women 15 - 49 years of age who are regular members of the selected households or who spent the night preceding the survey in the selected household. Eligible respondents were identified by using data on sex and age from the household questionnaire. The exclusion criteria was women of the same age group living in boarding schools or other collective housing units such as hotels, hospitals, prison and working accommodations (15). Data was collected through individual interviews with the women using standardized questionnaires administered by field staff trained on interview for data collection for this survey. A total of 12,481 women were identified for individual interviews. However, 11,865 women completed the questionnaires, producing a $95 \%$ response rate. Data entry was done using CSPro software for editing and processing (15).

\section{Measures}

\section{Outcome}

The outcome variable in this study was 'health care utilization' defined as women having visited a health facility for care during the last 12 months. This data was collected using the Gambia DHS $2019-20$ women's questionnaire through the question 'In the last 12 months, have you visited a health facility for care for yourself (or your children)? The response to this question was binary either yes or no. If a woman responded "Yes", it is recorded ' 0 ' and considered having utilized health care in the last 12 months, whiles if the woman responds to the question by saying "No" it is then recorded as ' 1 ' and considered as not having utilized health care in the last 12 months (15).

\section{Exposure}


The four main exposure or independent variables in this study related to problems accessing health care are: 1) permission to go for treatment, 2) getting money needed for treatment, 3 ) distance to health facility and 4) not wanting to go alone. These independent variables were all binary and coded as $1=$ big problem and $2=$ not a big problem in the analysis. Women were asked if each of these four factors would be considered a big problem creating a barrier to utilized health care for themselves or their children (15). The women respond "big problem" or "not a big problem" to any of these four variables as important for utilizing health care.

\section{Covariates}

Other demographic and socio-economic variables included in this study were individual demographic information such as age, educational attainment, residence, occupation, and household wealth quintile. These covariates are categorised in the following manner, Age group was categorised into 3 groups: $15-$ 24, 25 - 34, and 35 - 49. Education was as well categorised into 4 categories: higher, secondary, primary, and no education. Residency was categorised into rural, and urban. Occupation:

professional/technical/managerial, Clerical/services, manual, Agriculture, not working/household/domestic. Wealth quintile was categorised into 5 categories: richest, richer, middle, poorer, and poorest.

\section{Data Analyses}

Descriptive statistical analysis (including frequencies and percentages (\%)) was carried out for all variables stratified according to rural/urban residency. An unadjusted bivariate logistic regression was carried out to determine the association between health care utilization as an outcome and the problems of accessing health care as independent variables along with socio demographic covariates stratified by residence. The strength of association was determined by odds ratio (OR), confidence interval $(\mathrm{Cl})$ and $p$ value $<0.05$. Finally, an adjusted multi variable logistic regression model was carried out which adjusted for socio demographic covariates stratified by residence. The multi variable logistic regression included all four main independent variables in the analysis to assess if the direction of association will change when adjusted. In the final analysis, the strength of association was determined by adjusted odd ratio (AOR), confidence interval $(\mathrm{Cl})$ and $p$ value $<0.05$ stratified by residence. All data analysis in this study is done using STATA version 16.1.

\section{Results}

Table 1 presents descriptive statistics for the variables in the analysis. Looking at health care utilization among women 15 - 49 years of age. Most women (81\%) reported having visited a health facility during the last 12 months. By residence, majority of the women in both rural (79\%) and urban (83\%) residence visited health facility in the last 12 months. 
Table 1

Distribution of outcome, exposure, and covariate variables of the study population by residence

\begin{tabular}{|c|c|c|c|}
\hline & All, n (\%) & Rural & Urban \\
\hline \multicolumn{4}{|l|}{ variables } \\
\hline Visited health facility last 12 months & & $\mathrm{n}(\%)$ & $\mathrm{n}(\%)$ \\
\hline No & $5,883(19)$ & $2,961(21)$ & $2,922(17)$ \\
\hline Yes & $25,782(81)$ & $11,320(79)$ & $14,462(83)$ \\
\hline \multicolumn{4}{|l|}{ Getting permission to go } \\
\hline Big Problem & $1,142(4)$ & $532(4)$ & $610(4)$ \\
\hline Not a Big Problem & $30,523(96)$ & $13,749(96)$ & $16,774(96)$ \\
\hline \multicolumn{4}{|l|}{ Getting money needed for treatment } \\
\hline Big problem & $11,274(36)$ & $3,754(26)$ & $7,520(43)$ \\
\hline Not a big problem & $20,391(64)$ & $10,527(74)$ & $9,864(57)$ \\
\hline \multicolumn{4}{|l|}{ Distance to health facility } \\
\hline Big problem & $10,630(34)$ & $2,475(17)$ & $8,155(47)$ \\
\hline Not a big problem & $21,035(66)$ & $11,806(83)$ & $9,229(53)$ \\
\hline \multicolumn{4}{|l|}{ Not wanting to go alone } \\
\hline Big problem & $4,196(13)$ & $1,127(8)$ & $3,069(18)$ \\
\hline Not a big problem & $27,469(87)$ & $13,154(92)$ & $14,315(82)$ \\
\hline \multicolumn{4}{|l|}{ Age } \\
\hline $15-24$ & $2,300(7)$ & $923(6)$ & $1,377(8)$ \\
\hline $25-34$ & $10,997(35)$ & $5,070(36)$ & $5,927(34)$ \\
\hline $35-49$ & $18,368(58)$ & $8,288(58)$ & $10,080(58)$ \\
\hline \multicolumn{4}{|l|}{ Education } \\
\hline No education & $20,205(64)$ & $7,190(50)$ & $13,015(75)$ \\
\hline Primary & $5,098(16)$ & 2,510 (18) & $2,588(15)$ \\
\hline Secondary & $670(2)$ & $591(4)$ & $79(0.4)$ \\
\hline Higher & $5,692(18)$ & 3,990 (28) & 1,702 (9.6) \\
\hline
\end{tabular}




\begin{tabular}{|llll|}
\hline & All, $\mathbf{n}(\%)$ & Rural & Urban \\
\hline Pro/tech/managerial & $950(3)$ & $702(5)$ & $248(1)$ \\
\hline Clerical/services & $743(2)$ & $589(4)$ & $154(1)$ \\
\hline Manual & $1,175(4)$ & $977(7)$ & $198(1)$ \\
\hline Agriculture & $21,920(69)$ & $8,617(60)$ & $13,303(77)$ \\
\hline Not work/hhold/domestic & $6,830(22)$ & $3,370(24)$ & $3,460(20)$ \\
\hline Household wealth quantile & & & \\
\hline Richest & $6,691(21)$ & $2,349(16)$ & $4,342(25)$ \\
\hline Richer & $4,391(14)$ & $3,959(28)$ & $432(2)$ \\
\hline Middle & $6,004(19)$ & $4,098(29)$ & $1,906(11)$ \\
\hline Poorer & $11,432(36)$ & $728(6)$ & $10,704(62)$ \\
\hline Poorest & $3,147(10)$ & $3,147(22)$ & $0(0)$ \\
\hline
\end{tabular}

$\mathrm{n}$ : total number, \%: percentage

With regards to problems to the utilization of health care among women living in different parts of The Gambia, only about $5 \%$ of women living in both rural and urban residence reported getting permission to go as a big problem. Whereas $26 \%$ of women in rural and $45 \%$ in urban residence mentioned getting money needed for treatment as big problem in health care utilization. Similarly, by residence $15 \%$ of women in rural and $49 \%$ in urban residence cited distance to health facility as a big problem to health care utilization. Not wanting to go alone was cited as a big problem for only $4 \%$ of women in rural and $16 \%$ in urban residence describe not wanting to go alone as a big problem.

Tables 2 and 3, present results from the stratified bivariate and multivariable regression analysis, respectively. For women living in urban residences, health care utilization had a statistically significant association with getting permission to go and not wanting to go alone ( $p$ value $<0.05$ ). Women in urban residence that reported permission as a big problem had (AOR $0.38,95 \% \mathrm{Cl}: 0.32-0.46$ ) lower odds of health care utilization. Women in rural residence that reported distance to health facility as a big problem had (AOR 1.19, 95\% Cl: $1.05-1.35$ ) higher odds of health care utilization. Women in urban residence that reported not wanting to go alone as a big problem had (AOR $0.85,95 \% \mathrm{Cl}$ : $0.76-0.96$ ) lower odds of health care utilization compared to women who did not have big problem. 
Table 2

logistic regression showing the relationship between health care utilization and all exposure variables

\begin{tabular}{|c|c|c|c|c|}
\hline Outcome: health facility visits & Rural Residence & & & \\
\hline Variables & OR $(95 \% \mathrm{Cl})$ & $P$ value & AOR (95\% Cl) & p-value \\
\hline \multicolumn{5}{|l|}{ Getting permission to go } \\
\hline Not a Big problem & 1 & & 1 & \\
\hline Big problem & $0.89(0.72-1.10)$ & 0.291 & $0.83(0.67-1.03)$ & 0.100 \\
\hline
\end{tabular}

\section{Getting money needed for treatment}

Not a Big problem

1

1

Big problem

$1.08(0.99-1.19) \quad 0.080$

$1.04(0.94-1.15)$

0.389

\section{Distance to health facility}

Not a Big problem 1

1

Big problem

\section{$1.20(1.08-1.34) \quad 0.001$}

$1.19(1.05-1.35) \quad 0.005$

\section{Not wanting to go alone}

Not a Big problem 1

1

Big problem

$1.10(0.94-1.28) \quad 0.202$

$1.02(0.86-1.21)$

0.756

Age

15-24

1

25-34

$1.01(0.83-1.23) \quad 0.889$

$35-49$

$0.54(0.44-0.65) \quad<0.001$

\section{Education}

Higher

1

Secondary

$1.79(1.38-2.31) \quad<0.001$

Primary

$1.15(1.01-1.30) \quad 0.031$

No Education

$0.88(0.80-0.97) \quad 0.013$

Occupation

Pro/tech/managerial

1

Clerical/services

1.40 (1.07-1.84) 0.013

Manual

$0.82(0.65-1.02) \quad 0.088$

Agriculture

$1.27(1.06-1.52) \quad 0.008$ 


\begin{tabular}{|lll|}
\hline Outcome: health facility visits & Rural Residence & \\
\hline Not working/hhold/domestic & $1.28(1.05-1.55)$ & 0.011 \\
\hline Household wealth quantile & & \\
\hline Richest & 1 & \\
\hline Richer & $0.93(0.82-1.05)$ & 0.290 \\
\hline Middle & $1.12(0.99-1.27)$ & 0.067 \\
\hline Poorer & $0.97(0.79-1.19)$ & 0.784 \\
\hline Poorest & $0.96(0.84-1.10)$ & 0.630 \\
\hline
\end{tabular}

OR: odds ratio, AOR: adjusted odds ratio, Cl: confidence interval, 1: reference category 
Table 3

Logistic regression showing the relationship between health care utilization and all exposure variables

\begin{tabular}{|c|c|c|c|c|}
\hline Health facility visit & Urban residence & & & \\
\hline Variables & OR $(95 \% \mathrm{Cl})$ & $P$ value & AOR $(95 \% \mathrm{Cl})$ & $\mathrm{p}$-value \\
\hline \multicolumn{5}{|l|}{ Permission to go } \\
\hline Not a big problem & 1 & & 1 & \\
\hline Big problem & $0.35(0.29-0.41)$ & $<0.001$ & $0.38(0.32-0.46)$ & $<0.001$ \\
\hline \multicolumn{5}{|c|}{ Money needed for treatment } \\
\hline Not a big problem & 1 & & 1 & \\
\hline Big problem & $0.87(0.80-0.94)$ & 0.001 & $0.99(0.90-1.08)$ & 0.855 \\
\hline \multicolumn{5}{|c|}{ Distance to health facility } \\
\hline Not a big problem & 1 & & 1 & \\
\hline Big problem & $0.88(0.81-0.95)$ & 0.003 & $0.98(0.89-1.07)$ & 0.682 \\
\hline \multicolumn{5}{|l|}{ Not wanting to go alone } \\
\hline Not a big problem & 1 & & 1 & \\
\hline Big problem & $0.74(0.67-0.81)$ & $<0.001$ & $0.85(0.76-0.96)$ & 0.008 \\
\hline \multicolumn{5}{|l|}{ Age } \\
\hline $15-24$ & 1 & & & \\
\hline $25-34$ & $0.73(0.60-0.89)$ & 0.002 & & \\
\hline $35-49$ & $0.43(0.36-0.52)$ & $<0.001$ & & \\
\hline \multicolumn{5}{|l|}{ Education } \\
\hline Higher Education & 1 & & & \\
\hline Secondary & $1.99(0.85-4.64)$ & 0.108 & & \\
\hline Primary & $0.85(0.72-1.02)$ & 0.085 & & \\
\hline No Education & $0.78(0.67-0.90)$ & 0.001 & & \\
\hline \multicolumn{5}{|l|}{ Occupation } \\
\hline Pro/tech/managerial & 1 & & & \\
\hline Clerical/services & $9.46(3.34-26.79)$ & $<0.001$ & & \\
\hline Manual & $3.08(1.67-5.67)$ & $<0.001$ & & \\
\hline
\end{tabular}




\begin{tabular}{|lll|}
\hline Health facility visit & Urban residence & \\
\hline Agriculture & $1.23(0.90-1.68)$ & 0.190 \\
\hline Not working/hhold/domestic & $1.24(0.89-1.71)$ & 0.190 \\
\hline Household wealth quintile & & \\
Richest & & \\
Richer & $0.63(0.48-0.81)$ & $<0.001$ \\
Middle & $0.66(0.57-0.77)$ & $<0.001$ \\
Poorer & $0.68(0.61-0.75)$ & $<0.001$ \\
\hline
\end{tabular}

OR: odds ratio, AOR: adjusted odds ratio, Cl: confidence interval, 1: reference category

\section{Discussion}

\section{MAIN FINDINGS}

The study indicated that health care utilization is high in The Gambia in both rural and urban residence. However, by residence the findings of the study showed, in urban residence the problems to health care utilization are permission to go and Not wanting to go alone. Whereas, in rural residence the association of getting permission to go as a big problem and health care utilization is not statistically significant.

\section{HEALTH CARE UTILIZATION}

The descriptive analyses of this study revealed high utilization of health care of about $80 \%$, by extend women of rural and urban residence report $77 \%$ and $81 \%$ of health care utilization, respectively. This could be attributed to the extensive national primary health care program in the Gambia which provided $85 \%$ of the population with access to primary health care within every 5 kilometres (17). Contrary to these findings, studies in Timor-Leste reports primary health care utilization is $27 \%$ higher in rural residence than urban residence. This can be associated with poorly develop infrastructure causing geographical inaccessibility, cultural barriers to health care seeking or high poverty level in rural residence.

\section{PERMISSION TO GO}

In urban residence women who had big problem getting permission to go are less likely to utilize health care compared to women who did not have a problem getting permission to go. However, in rural, residence getting permission to go is not associated with health care utilization for women who considered it big problem. This finding suggests that getting permission to go is a barrier to women in urban residence using health care. Contrary to this findings, similar studies in Ghana reported permission to go being a barrier to health care access is equal in both rural and urban residence (14). 
In rural residence, women who had big problem getting money needed for treatment were more likely to utilize health care compared to women who did not have a big problem getting money needed for treatment. Reasoning to this, money needed for treatment is not a barrier to health care utilization among rural women who consider it to be big problem. In contrast, in urban residence women who had big problem getting money needed for treatment are less likely to utilize health care compared to women who did not have a big problem getting money needed for treatment. This explains that getting money needed for treatment is a barrier to health care utilization for women in urban residence as it is militating women using health care. The association of money needed for treatment and health care utilization is not statistically significant in both rural and urban residence. Contrary to this finding, a study of health care utilization and its barriers in Kenya after the introduction of free maternity service suggests that poor women in remotes slums are challenge with buying medications leading to low utilization of health services in rural Kenya $(11,18)$. Women in rural residence are to pay for consultation and other incidental fees of cost recovery for gloves and other routine materials needed for the maintenance of health services (19).

\section{DISTANCE TO HEALTH FACILITY}

In rural residence women who had big problem concerning long distance to health facility were more likely to utilized health care compared to those who did not have big problem concerning long distance to health facility. Whereas, in urban residence women who had big problem concerning long distance to health facility were less likely to utilized health care compared to those who did not consider long distance to health facility a big problem. However, the association of distance to health facility with health care utilization is only statistically significant in rural residence. This could be associated with the expansion of primary health care in The Gambia reaching $97 \%$ and $85 \%$ of the population living within 5 and 3 kilometres, respectively from a primary health care post (20). This finding is in disagreement with 2014 DHS in Ghana which reported women in rural residence have $41 \%$ higher chance of distance being a barrier to health care utilization compared to urban residence (14).

\section{NOT WANTING TO GO ALONE}

In rural residence, women who had big problem concerning not wanting to go alone for health care utilization were more likely to utilized health care compared to those who did not have a big problem not wanting to go alone. Whereas, in urban residence women who had big problem concerning not wanting to go alone for health care utilization were less likely to utilized health care compared to those who did not have a big problem not wanting to go alone. However, not wanting to go alone is associated with health care utilization in urban residence only. This could be attributed to weak culture of social support and companionship in urban residence where women live in small family size. Similar studies in Tanzania reported not wanting to go alone having $4 \%$ higher chance of being a big problem for women compared to not a big problem for women (21). More studies could not be found highlighting the disparity of not wanting to go alone as a big problem for women utilization of health care by residence. 
The findings from this study revealed some problems to health care utilization in The Gambia. As The Gambia is advancing towards universal health coverage, policies and programmes are recommended to improve health care utilization in urban residence. Furthermore, in order to improve equity and access to health care for the attainment of the Universal Health Coverage goals, social programmes targeting companionship during health care seeking should be implemented to improve health care utilization in urban residence. Further studies are recommended to explore the disparities of health care utilization by residence and its determinants to provide evidence to improve access and equity in health care utilization.

\section{STRENGTHS AND LIMITATIONS}

In one hand, this study used secondary data from the 2019 - 20 Gambia DHS which entailed a nationally representative sample which increase the generalizability of the finding. The definition of the outcome variable as the utilization of health care in the last 12 months and problems associated with it in the last 12 months reduces recall bias of the study participants.

On the other hand, cross sectional studies cannot establish causality and this study did not consider other factors which may influence health care utilization such as the need for health care. Further, this study could not ensure women who responded, "Not a big problem" and "Big problem" to the problems of health care utilization have the same need for care.

\section{Conclusion}

Some problems to health care utilization in The Gambia is evident in urban residence. The problems to health care utilization appear to be associated with permission to go and not wanting to go alone in urban residence because of the weak culture of social support and close family networks supporting women when seeking health care.

\section{Abbreviations}

AOR

adjusted odds ratio

$\mathrm{Cl}$

Confidence Interval

DHS

Demographic and Health Survey

GDP

Gross Domestic Product

ICF

Inner-City Fund International

IRBs 
Institutional Review Boards

MRC

Medical Research Council

OR

odds ratio

SDG

Sustainable Development Goals

$\mathrm{UHC}$

Universal Health Coverage.

\section{Declarations}

\section{AVAILABILITY OF DATA AND MATERIALS}

The data and analysis supporting the findings of this study can be made available by the corresponding author upon request. The data set used for this research is publicly available at MEASURE DHS via : https://www.dhsprogram.com/data/dataset

\section{ETHICS APPROVAL AND CONSENT TO PARTICIATE}

Ethical approval for The Gambia 2019 - 20 DHS was provided by The Gambia Government/Medical Research Council (MRC) Joint Ethics Committee in The Gambia and the Institutional Review Boards (IRBs) at Inner-City Fund International (ICF), USA. The protocol for the survey methodology was approved by both institutional review boards prior to the data collection process (15). The data collection was performed in accordance with ethical guidelines and regulations of these institutional review boards. Inform consent was sought from the respondents and parents or guidance of teenage girls $15-17$ years in which they had the opportunity either not to participate or drop out of the interview at any time (15). The data set used for this research paper is publicly available therefore ethical approval for this study was not necessary.

\section{CONSENT FOR PUBLICATION}

Not Applicable

\section{AVAILABILITY OF DATA AND MATERIALS}

The authors analysed secondary data in this study which can be access at: The DHS Program login_main

\section{COMPETING INTEREST}

The authors declare that they have no competing interest. 
This study did not benefit from any source of funding.

\section{AUTHORS' CONTRIBUTIONS}

EB and AS design the study; EB did the data analysis, and interpretation of data. EB and AS drafted and reviewed the manuscript. All authors read and approved the final manuscript.

\section{ACKNOWLEDGEMENTS}

We would like to thank Umeå university for creating the conducive environment for research, EB is grateful to the Swedish Institute for providing the scholarship to pursue graduate studies in Sweden. We acknowledge the support of Frida Jonsson during the design of the research. Special gratitude to DHS program for giving us access to the survey data sets

\section{AUTHORS' INFORMATION}

$\mathrm{EB}^{1}$ : Public Health Officer, Ministry of Health, The Gambia. AS ${ }^{2}$ : Public Health Officer, Ministry of Health, The Gambia.

\section{References}

1. Carrasquillo O. (2013) Health Care Utilization. In: Gellman M.D., Turner J.R. (eds) Encyclopedia of Behavioral Medicine. Springer, New York, NY. https://doi.org/10.1007/978-1-4419-1005-9_885. Accessed 24 April 2021.

2. Andersen newman framework.1995 https://umanitoba.ca/.../protocol/media/Andersen_and_Newman_Fra... P PDF file. Accessed 24 April 2021.

3. Andersen RM. Revisiting the Behavioral Model and Access to Medical Care: Does it Matter? J Health Soc Behav. 1995;36(1):1-10.

4. Starrs AM, Ezeh AC, Barker G, Basu A, Bertrand JT, Blum R, et al. Accelerate progress-sexual and reproductive health and rights for all: report of the Guttmacher- Lancet Commission. The Lancet. 2018 Jun;391(10140):2642-92.

5. Hogan DR, Stevens GA, Hosseinpoor AR, Boerma T. Monitoring universal health coverage within the Sustainable Development Goals: development and baseline data for an index of essential health services. Lancet Glob Health. 2018 Feb;6(2):e152-68.

6. United Nations, Department of Economic and Social Affairs, sustainable development goals. https://sdgs.un.org/goals/goal3. Accessed 28 April 2021.

7. Breaking the barriers towrads more equitable and gender responsive health systems WHO 2019. . Accessed 1 May 2021.

8. Bodily autonomy is a fundamental human right [Internet]. UNFPA - United Nations Population Fund. [cited 2021 May 1]. Available from: https://www.unfpa.org/sowp-2021. Accessed 1 May 2021 
9. Tsawe M, Susuman AS. Determinants of access to and use of maternal health care services in the Eastern Cape, South Africa: a quantitative and qualitative investigation. BMC Res Notes. 2014 Oct 15;7(1):723.

10. Munguambe K, Boene $H$, Vidler M, Bique $C$, Sawchuck D, Firoz T, et al. Barriers and facilitators to health care seeking behaviours in pregnancy in rural communities of southern Mozambique. Reprod Health. 2016 Jun;13(S1):31.

11. Ochieng CA, Odhiambo AS. Barriers to formal health care seeking during pregnancy, childbirth and postnatal period: a qualitative study in Siaya County in rural Kenya. BMC Pregnancy Childbirth. 2019 Dec;19(1):339.

12. Ganle JK, Parker M, Fitzpatrick R, Otupiri E. A qualitative study of health system barriers to accessibility and utilization of maternal and newborn healthcare services in Ghana after user-fee abolition. BMC Pregnancy Childbirth [Internet]. 2014 Dec 21 [cited 2021 May 2];14. Available from: https://www.ncbi.nlm.nih.gov/pmc/articles/PMC4307897/. Accessed 2 May 2021

13. The Gambia Bureau of Statistics (GBOS) and ICF International. 2014. The Gambia Demographic and Health Survey 2013. Banjul, The Gambia, and Rockville, Maryland, USA: GBOS and ICF International.

14. Seidu A-A, Darteh EKM, Agbaglo E, Dadzie LK, Ahinkorah BO, Ameyaw EK, et al. Barriers to accessing healthcare among women in Ghana: a multilevel modelling. BMC Public Health. 2020 Dec;20(1):1916.

15. Gambia Bureau of Statistics (GBoS)and ICF. 2021. The Gambia Demographic and Health Survey 2019-20. Banjul, The Gambia and Rockville, Maryland, USA: GBoS and ICF.

16. MEASURE DHS https://www.dhsprogram.com/data/dataset.

17. Gambia national health sector strategic plan 2014-2020.

18. Masaba BB, Mmusi-Phetoe RM. Free Maternal Health Care Policy in Kenya; Level of Utilization and Barriers. Int J Afr Nurs Sci. 2020 Jan 1;13:100234.

19. Klein MC, Harvey SA, Diarra H, Hurley EA, Rao N, Diop S, et al. "There is no free here, you have to pay": actual and perceived costs as barriers to intermittent preventive treatment of malaria in pregnancy in Mali. Malar J. 2016 Mar 12;15(1):158.

20. 2012 - Gambia - Country Gender Profile.:56.

21. Gupta S, Yamada G, Mpembeni R, Frumence G, Callaghan-Koru JA, Stevenson R, et al. Factors Associated with Four or More Antenatal Care Visits and Its Decline among Pregnant Women in Tanzania between 1999 and 2010. PLOS ONE. 2014 Jul 18;9(7):e101893. 